\title{
Internally-Frequency-Doubled Yb Fiber Laser with 15 W CW Green Output
}

\author{
R. Cieslak and W. A. Clarkson \\ Optoelectronics Research Centre, University of Southampton, Southampton, S017 1BJ, UK \\ Author e-mail address: rxc@orc.soton.ac.uk
}

In this paper we present a high power continuous-wave $(\mathrm{CW})$ green source using a novel approach for frequency doubling of high power CW fiber lasers based on an internal resonant enhancement cavity integrated within the fiber laser resonator. The experimental configuration (shown in Fig. 1) comprised a double-clad fiber with an $\mathrm{Yb}$-doped core in a simple standing-wave resonator. Feedback for lasing was provided by a diffraction grating at one end of the fiber, and by an external cavity containing a resonant enhancement cavity at the opposite end of the fiber. The enhancement cavity comprised a Brewster-angled $\mathrm{LiB}_{3} \mathrm{O}_{5}(\mathrm{LBO})$ crystal placed in an oven and cut for type I non-critical phase-matching. In this scheme, the fiber laser automatically lases on axial modes that are resonant in the enhancement cavity avoiding the need for active stabilisation. The fundamental laser power is enhanced in the resonant cavity by virtue of its relatively low loss yielding high second harmonic conversion efficiency.

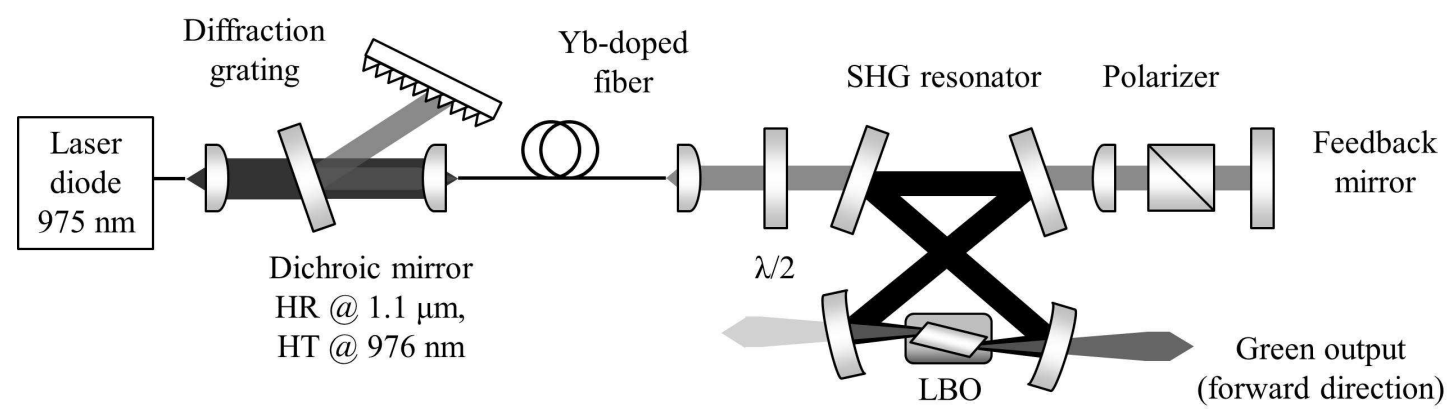

Fig. 1. Experimental set-up for internal frequency doubling in a fiber laser

With this setup, we obtained $15 \mathrm{~W}$ of $\mathrm{CW}$ second harmonic output (at $540 \mathrm{~nm}$ ) in the forward direction (Fig. 1), corresponding to $19 \mathrm{~W}$ generated inside the LBO crystal, for $90 \mathrm{~W}$ of absorbed diode pump power (at $975 \mathrm{~nm}$ ). The output power in the reverse direction was $<100 \mathrm{~mW}$. The internal conversion efficiency of the resonator with respect to the fundamental power entering the enhancement cavity was $>63 \%$. The output was linearly-polarized and its beam quality factor $\left(\mathrm{M}^{2}\right)$ was measured to be $<1.25$. The laser was tunable over the range of 540-560 nm (for $20 \mathrm{~m}$ long fiber) and over the range 520-550 nm (for $10 \mathrm{~m}$ long fiber) by adjusting the grating angle and the oven temperature to maintain phase matching. These preliminary results were obtained with a non-optimal set-up due to limited availability of components. The prospects for further improvement in performance in terms of output power and range of operating wavelengths will be discussed.

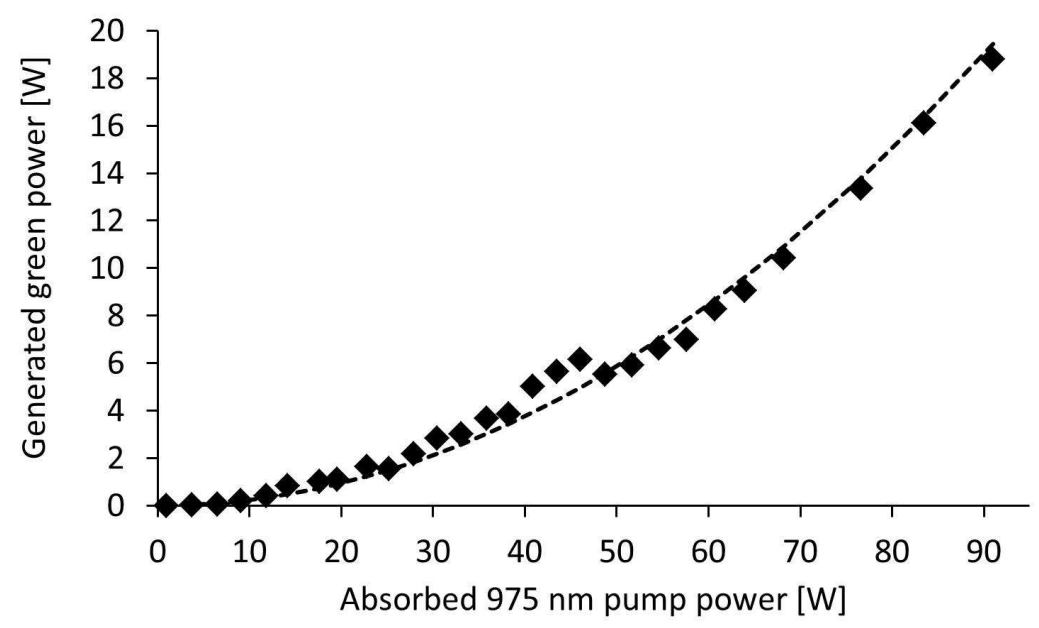

Fig. 2. Generated second harmonic power at $540 \mathrm{~nm}$ as a function of absorbed pump power at $975 \mathrm{~nm}$ 